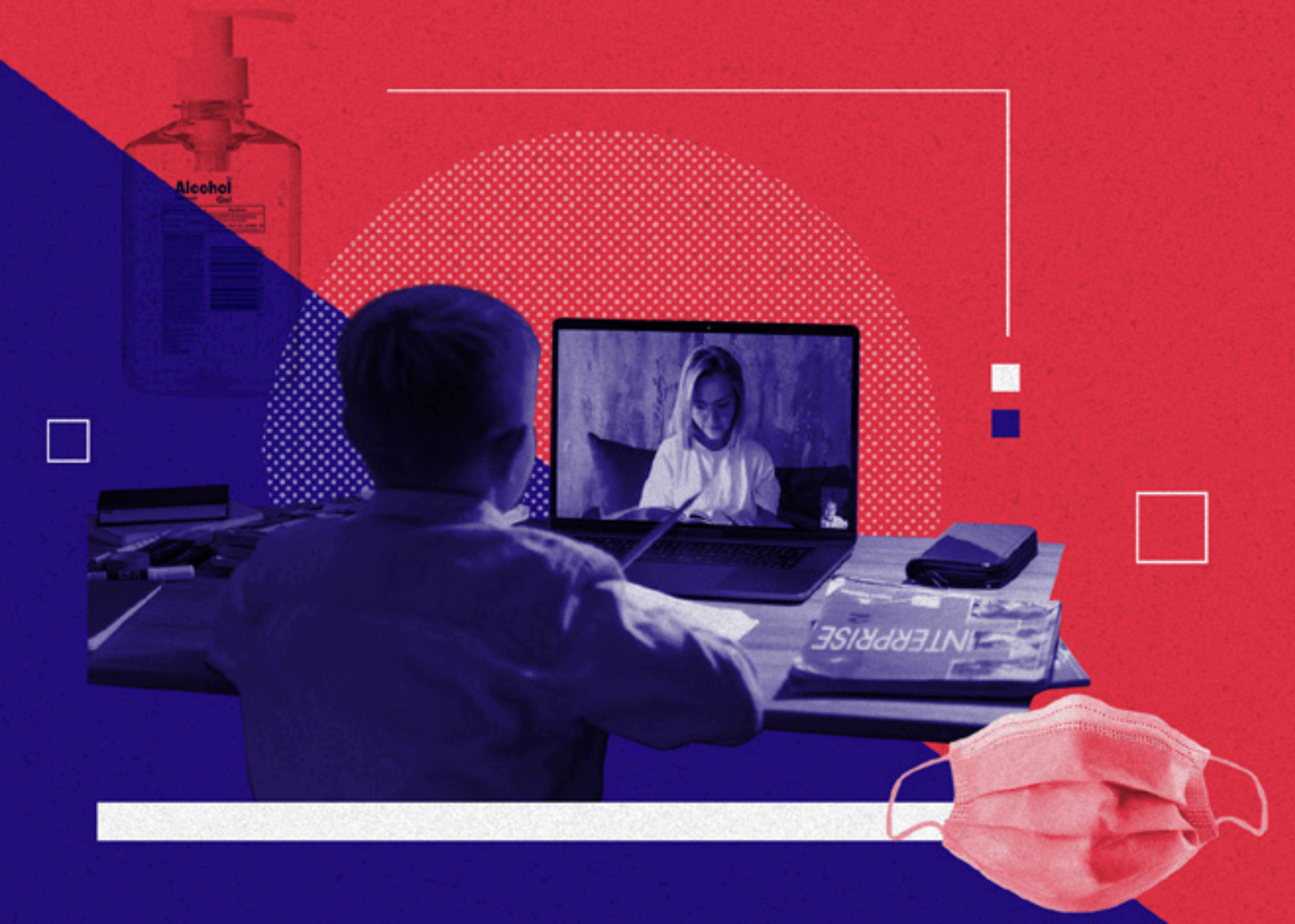

\title{
La educación, persistencias, transfiguraciones y desvanecimientos en tiempos de pandemia
}

EDUCATION, PERSISTENCE, TRANSFIGURATION, AND VANISHING IN TIMES OF PANDEMIC

EDUCAÇÃO, PERSISTÊNCIA, TRANSFIGURAÇÃO E DESAPARECIMENTO EM TEMPOS DE PANDEMIA

Óscar Leonardo Cárdenas Forero; María Liliana Benítez Agudelo²; Sonia Milena Uribe Garzón ${ }^{3}$ 
Citar artículo como:

Cárdenas-Forero, O.L, Benítez-Agudelo, M.L., y Uribe-Garzón, S.M. (2021). La educación, persistencias, transfiguraciones y desvanecimientos en tiempos de pandemia. Educación y Ciudad, n. 41, pp. 211 - 228. https://doi.org/10.36737/01230425.n41.2021.2520

Fecha de recepción: 8 de abril de 2021

Fecha de aprobación: 6 de julio de 2021

\section{Resumen}

El presente artículo describe las persistencias, transfiguraciones y desvanecimientos que han enfrentado las experiencias pedagógicas de algunos maestros en tiempos de pandemia. Para ello, se muestran 3 prácticas adelantadas por estos maestros en instituciones educativas públicas. Entre los hallazgos, están las nuevas relaciones con el conocimiento, el aprendizaje y el desarrollo, incorporándose los medios digitales como estrategia de continuidad a las prácticas de enseñanza. En conclusión, dichas prácticas se reconfiguraron, adaptaron y dispusieron de un modo distinto para mantenerse activas.

Palabras clave: Educación; pandemia; persistencias; transfiguraciones; desvanecimientos.

\begin{abstract}
This article describes the persistence, transfiguration, and fading that the pedagogical experiences which some teachers have faced in times of pandemic. To carry out this, 3 practices carried out by these teachers in public educational institutions are particularly shown. Among the findings, new relationships were established with knowledge, learning and development, incorporating digital media as a strategy for continuity in teaching practices. In conclusion, these practices were reconfigured, adapted, and arranged in a different way to stay active in time.
\end{abstract}

Keywords: Education; pandemic; persistence; transfiguration; vanishing.

\section{Resumo}

Este artigo descreve a persistência, transfiguração e esmaecimento que as experiências pedagógicas dos professores enfrentaram em tempos de pandemia. Para tanto, são apresentadas três práticas realizadas por professores em instituições públicas de ensino. Entre as constatações estão que, diante dessa situação emergente, novas relações se estabeleceram com o conhecimento, a aprendizagem e o desenvolvimento, incorporando as mídias digitais como estratégia de continuidade das práticas docentes. Em conclusão, essas práticas foram reconfiguradas, adaptadas e organizadas de uma maneira diferente para permanecerem ativas no tempo.

Palavras-chave: Educação; pandemia; persistência; transfiguração; desaparecimento. 


\section{Introducción}

工

a situación de confinamiento que se vivió en 2020 provocó la transformación de las condiciones históricas del planeta,

[...] no solo en la forma de comprender el mundo, en su tejido social, en los juegos del poder, en las relaciones económicas y culturales, en la esfera de las ciencias, en la fragilidad del acceso y dominio del conocimiento, sino en las diferentes formas de comunicación, y por supuesto, en la estructura visible y enunciable de la educación y la escuela (Maestros en Colectivo, 2021, p. 8).

Por esta razón, sectores como la educación y la escuela se vieron enfrentadas a la alteración de sus modos de funcionamiento, a la transfiguración de las maneras de enseñar y a la adopción de medios alternativos para la transmisión de la información, cuyo propósito esencial era el de brindar continuidad a la formación y a los procesos de aprendizaje de los niños, niñas y jóvenes. Los países, según el Informe Cepal-Unesco (2020) sobre COVID-19, suspendieron las clases presenciales en todos los niveles, generando:

Docente Colegio Entre Nubes S.O. Magíster en Desarrollo Educativo y Social de la Universidad Pedagógica Nacional -CINDE. ORCID: http://orcid.org/0000-0003-48947888 Correo electrónico: olcardenasf@ut.edu.co

Docente Colegio Instituto Técnico Industrial Piloto. Especialista en Pedagogía de la Lúdica, Universidad Los Libertadores. ORCID: https://orcid.org/0000-0002-40227918 Correo electrónico: tomalibea@gmail.com
[...] el despliegue de modalidades de aprendizaje a distancia, mediante la utilización de una diversidad de formatos y plataformas (con o sin uso de tecnología); el apoyo y la movilización del personal y las comunidades educativas, y la atención a la salud y el bienestar integral de las y los estudiantes (p. 1).

Esto implicó no solo que se afectara “[...] la alimentación y la nutrición de la población estudiantil, especialmente en los sectores más vulnerables" (p. 2), sino que los maestros se vieran abocados a incluir, de una manera más marcada, los instrumentos y mediaciones virtuales para garantizar la educación. Al punto, que los procesos educativos transitaron hacia los hogares de los maestros y estudiantes, posibilitando la irrupción de las "modalidades de aprendizaje a distancia en línea" y el uso de "plataformas virtuales de aprendizaje asincrónico", y procurando mantener el contacto, la comunicación y la continuidad de la enseñanza, de los aprendizajes y de los desarrollos ${ }^{4}$.

Junto con esto, el mundo digital y sus herramientas incursionaron como alternativas para complementar, apoyar y garantizar, precisamente, la continua-

Docente Colegio Entre Nubes S. O., Magíster en Desarrollo Educativo y Social de la Universidad Pedagógica Nacional CINDE. ORCID: http://orcid.org/0000-0002-8401-1780 Correo electrónico: sonia.smug80@gmail.com

Según Becerra et. al. (2021) "[...] la metodología de enseñanza en el país se reconfiguró, al pasar de la enseñanza presencial a la implementación de estrategias instruccionales de carácter virtual o de educación en casa usando como mediaciones la televisión, radio y, por supuesto, los diseños de actividades de aprendizaje elaboradas por los maestros" (p. 106). 
ción de la educación en casa, transformando los discursos y prácticas pedagógicas, en la medida en que, el quehacer del maestro se vio invadido por el uso de nuevas acciones de intervención educativa virtual, el manejo de un lenguaje tecnológico y de unos modos específicos de actuación en relación con la virtualidad. Prueba de ello, fue el diseño e implementación, a nivel nacional, y por supuesto, en Bogotá, de la estrategia "Aprende en casa", una acción educativa dirigida a dar continuidad al ejercicio formativo, al aprendizaje mediado y a la conversión del hogar en un ambiente de aprendizaje ${ }^{5}$.

De esa manera, se hizo cotidiano "el trabajo a distancia” y conectarse a través de Zoom, Google Meet o Teams, entre otras plataformas para asegurar la educación, lo que transformó las formas de ser sujetos, la enseñanza, el aprendizaje, las relaciones de poder, los comportamientos y maneras de interactuar con los demás, constituyéndose un nuevo "ambiente de aprendizaje" ${ }^{6}$, en el que las "herramientas útiles y nuevas formas de comunicación con los alumnos y la comunidad educativa" (Organización de las Naciones Unidas, 2020, pp. 16-17), se convirtieron en los ejes dinamizadores de su acontecer.

Para profundizar sobre la estrategia "Aprende en casa" se puede consultar: https://www.redacademica.edu.co/estrategias/aprende-en-casa Directiva No. 5 del 25 de marzo de 2020

En este ambiente de aprendizaje emergente, las relaciones personales se vieron afectadas en gran medida, ya que, del contacto cotidiano, físico y directo entre las personas, se gestó el tránsito al establecimiento de unas relaciones a distancia, mediadas por la pantalla y el alejamiento, haciendo de la construcción de lo social y de las relaciones de poder, un asunto de mediación virtual.
Esto significo que las experiencias de los maestros tuvieron que flexibilizar sus propósitos, realizar ajustes a los contenidos de enseñanza, a las metodologías, a los recursos didácticos y a las formas de evaluar los aprendizajes y desarrollos de los estudiantes; asociado a la aplicación de una serie de "[...] enfoques innovadores en apoyo de la continuidad de la educación y la formación, recurriendo a la radio y la televisión y a materiales para llevar" (Naciones Unidas, 2020, p. 2).

Pero, además, que se alterara la manera cómo se venía ejecutando el currículo "[...] no solo por el uso de plataformas y la necesidad de considerar condiciones diferentes a aquellas para las cuales el currículo fue diseñado, sino también porque existen aprendizajes y competencias que cobran mayor relevancia en el actual contexto" (Cepal-Unesco, 2020, p. 4). Todo ello, derivó en que las prácticas de enseñanza adquirieran un nuevo formato, con el que se buscó trascender las barreras impuestas por el distanciamiento al que se enfrentaban maestros y estudiantes en esta racionalidad floreciente ${ }^{7}$. Y con ello, que se resaltara la importancia del maestro y de la escuela en el desarrollo educativo y en la forma-

Según la Cepal-Unesco (2020) "La mayoría del magisterio no solo ha debido replanificar y adaptar los procesos educativos, lo que incluye ajustes de metodología, reorganización curricular, diseño de materiales y diversificación de los medios, formatos y plataformas de trabajo, entre otros aspectos, sino que también ha debido colaborar en actividades orientadas a asegurar condiciones de seguridad material de las y los estudiantes y sus familias, como la distribución de alimentos, productos sanitarios y materiales escolares, entre otros. El profesorado y el personal educativo ha debido enfrentar las demandas de apoyo socioemocional y de salud mental de las y los estudiantes y sus familias, dimensión que ha cobrado creciente relevancia durante la pandemia" (p. 10). 
ción de los estudiantes, en la medida, en que, a pesar de las circunstancias de la pandemia, la producción del saber pedagógico y escolar no se detuvo, sino que, por el contrario, se transfiguró y continúo circulando por otros medios.

Igualmente, comenzaron a irrumpir discursos que señalaron que esta era la oportunidad para "transformar a la escuela" con el fin de insertarla en el mundo virtual, realizando "una transformación desde sus bases", para lo cual, no bastaba con implementar recursos tecnológicos, sino que era necesario reinventar la escuela y realizar las reformas necesarias para una efectiva cultura digital (Hurtado, 2020). Sin embargo, más allá de estas pretensiones, lo que se manifestó fue que el trabajo del maestro y el espacio escolar, eran componentes esenciales en el desarrollo, en la construcción de conocimiento y en la formación subjetiva.

Como se observa, la intrusión de esta nueva condición social trajo como consecuencia, que algunas experiencias de aula, en particular, aquellas con pretensiones de "innovar", se fortalecieran en este escenario virtual, pues incorporaron las mediciones en entornos digitales para el ejercicio pedagógico y la consecución de los objetivos de aprendizaje establecidos. Otras, en cambio, (en especial, aquellas que requirieron de la presencialidad para su ejecución), sencillamente optaron por diluirse, ante la imposibilidad de continuar con sus intenciones en un escenario de virtualización de la enseñanza.

No solo los maestros se vieron comprometidos a incluir en sus prácticas de enseñanza los dispositivos tecnológicos y una serie de estrategias digitales sino que tuvieron que, además de flexibilizar y ajustar contenidos, objetivos, metodologías y formas de evaluación, diseñar un conjunto de actividades de enseñanza que se materializaron en módulos y cartillas de trabajo, que los niños debieron desarrollar en casa, con el acompañamiento constante de los padres de familia, acudientes y cuidadores; así, se pretendió continuar y complementar la formación educativa de los estudiantes.

Para dar cuenta de estas transformaciones, permanencias y desvanecimientos en tiempos de pandemia, a continuación, se describen 3 experiencias pedagógicas que se llevaron a cabo en 2 instituciones educativas en Bogotá. La primera de ellas, " $L a$ cartilla pedagógica”, una propuesta metodológica y didáctica enmarcada en la integración curricular de los campos de pensamiento para la enseñanza y el aprendizaje del lenguaje. La segunda, "Las guías integradas", una estrategia colegiada que se materializó en una cartilla pedagógica, que se dirigió a los aprendizajes esenciales de los estudiantes, en el marco de cada una de las áreas académicas. Y la tercera, " $L a$ abstracción geométrica", una estrategia didáctica para el desarrollo del pensamiento geométrico de los niños en la escuela primaria, que se mantuvo en el escenario digital. Es precisamente, a través de cada una de estas vivencias pedagógicas, que se buscan relatar las condiciones a las que se enfrentó el trabajo de los maestros durante la pandemia.

\section{La cartilla pedagógica}

Durante mucho tiempo, la escuela se ha constituido en el escenario fundamental en el que el maestro, en el reflexionar sobre su accionar pedagógico, se centra, entre otras cosas, en cómo abordar la enseñanza de una serie de contenidos programáticos seleccionados y prescritos muchas veces por agentes externos a la escuela, y otras, formulados por el mismo maestro conforme con las condiciones contex- 
tuales de la misma, para transmitirlos y llevarlos a los estudiantes, de manera que estos contenidos de enseñanza, adquieran sentido y significación tanto para el sujeto que los enseña como para quienes lo aprenden. Esta reflexión constante, es la que les permite a los maestros otorgarle sentido a la búsqueda de rutas, mecanismos y estrategias diferentes para abordar tanto los aspectos metodológicos como didácticos que constituyen la enseñanza escolar, y que le permiten diseñar y ejecutar acciones de intervención frente a situaciones observadas, propias del acontecer escolar.

Esta actitud pedagógica, durante la pandemia no se resquebrajó, desapareció o desdibujó. Al contrario, se mantuvo constante entre muchos de los maestros. Y en particular, el cuestionamiento sobre ¿Cómo dar continuidad a la enseñanza, al desarrollo de los aprendizajes y al camino que se debía seguir para mantener la formación educativa? Una pregunta asumida por el grupo de maestras del grado primero de la jornada mañana, del colegio Instituto Técnico Industrial Piloto, que hicieron factible la reflexión sobre cuál de las múltiples posibilidades era la más pertinente para dar continuidad a los procesos educativos de los niños y niñas.

Esto significó construir aquella ruta metodológica y didáctica que diera respuesta tanto a las exigencias

\footnotetext{
El trabajo sobre los campos de pensamiento, en el marco de la teoría de la complejidad de Edgar Morin, fue una propuesta de la Secretaría de Educación del Distrito (SED) que formuló: "A cambio de un currículo prediseñado por áreas, asignaturas y resultados de aprendizaje, se ha propuesto la discusión de campos de pensamiento complejo, que permiten ver la interrelación de perspectivas diversas
}

contextuales de pandemia como a las institucionales frente al grado primero, así como contemplar el interés de las maestras por darle continuidad a la reflexión sobre ¿Qué se enseña?, ¿para qué se enseña? y ¿cómo se enseña?, además de considerar en ese ejercicio, las necesidades, requerimientos y expectativas de los estudiantes frente a la comunicación. De allí que se estableció como premisa entre las maestras, que para asumir los retos de la pandemia y abordar estas cuestiones pedagógicas, era primordial reconocer que la oralidad, la lectura y la escritura en el grado primero, eran lo esencial a desarrollar en casa con el acompañamiento de los padres de familia. En definitiva, estos aspectos comunicativos se constituyeron en el eje transversal para el marco de los campos de pensamiento ${ }^{8}$, así como para la construcción del conocimiento y el desarrollo de los demás aprendizajes en niños y niñas.

Dicho lo anterior, una vez inmersas en la condición de pandemia, se acordó entre las maestras continuar con la propuesta metodológica y didáctica enmarcada en la integración curricular de los campos de pensamiento para la enseñanza y el aprendizaje del lenguaje, formulada antes de la pandemia, que combinó teorías globales y sintéticas, características de la lectoescritura y las necesidades, intereses y contextos de los niños y niñas, no sin antes, realizar un proceso de reflexión, análisis y adaptación conforme a la nueva realidad.

cuando se aborda la reflexión sobre los fenómenos del mundo. Se trata de introducir una profunda ruptura epistemológica, que, de prioridad al aprendizaje como proceso de reflexión permanente sobre la experiencia cognitiva, en vez de centrarse sobre la organización secuencial de información fragmentada por disciplinas con el fin de facilitar la enseñanza y la homogenización" (SED, 2007, p. 16). 
Esto trajo como consecuencia, asumir la situación de aislamiento como una oportunidad para darle continuidad al trabajo iniciado, y en especial, el de los campos de pensamiento, solamente que esta vez, debía articularse a la perspectiva de la estrategia de la SED "Aprende en casa". Un desafío que se tradujo en un proceso de reconfiguración, que comprendió la necesidad de destacar el valor de estar con el otro, por medio de las herramientas digitales, para garantizar tanto la enseñanza -propósito del trabajo del equipo de maestras- como el aprendizaje de los niños- que se procuró fuera significativo-.

Esta situación fue inicialmente resuelta con la producción de una serie de guías de trabajo, que con el transcurrir de los días, llegaron a estar en contravía, contradiciendo y desdibujando las intenciones que previamente, en la presencialidad se habían formulado y definido.

Ante estas circunstancias, surgió la pregunta reflexiva de ¿Cómo organizar una ruta de trabajo mediada por el escenario digital, que "realmente" responda a las expectativas e intereses de los estudiantes y maestras? Algo que lleva a pensar en la igualdad de condiciones respecto al acceso a la educación como derecho, y que, a su vez, facilite y guie a los padres de familia, cuidadores y acudientes en el ejercicio de acompañamiento, para lograr con esto, la promoción y desarrollo de unas capacidades, habilidades, destrezas y conocimientos en esta nueva cotidianidad, sin abandonar, por supuesto, la ruta metodológica y didáctica que se estableció a comienzos del año.

Justamente, es a raíz de esta pregunta tan compleja y retadora, que se elaboró un material pedagógico centrado en una planificación secuencial, progresiva y contextualizada, que se relacionó con las intenciones de conocimiento plasmadas en el currículo institucional, y que se acopló con la propuesta de los campos de pensamiento, lo que facilitó la flexibilidad, la eficacia, el acceso y el apoyo familiar en el proceso educativo, así como la introducción de acciones integrales, reflexivas y trascendentes (Figura 1).

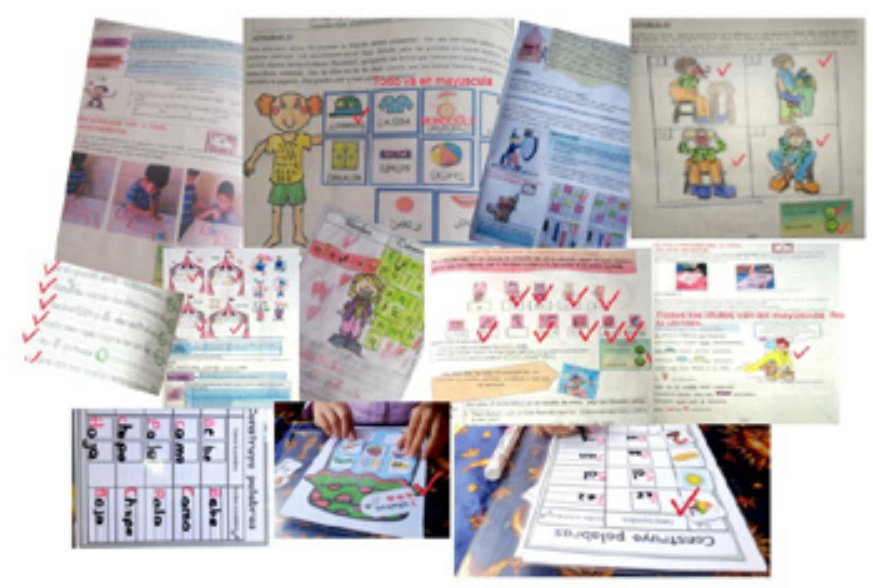

Figura 1. Evidencias de las acciones de los niños Fuente: Archivo personal de los autores.

Esta propuesta se enfoca en el diseño y ejecución de una unidad didáctica integrada (Figura 2), basada en una serie de relatos asociados a la vida en el circo, que sirvió de referente para proponer las actividades de enseñanza y se materializó en el diseño de una cartilla pedagógica (Figura 3), compuesta por una conjunción de actividades y ejercicios que les permitieron a los niños avanzar en los procesos de aprendizaje y conocimiento pertinentes para el grado primero. 
Tabla 1. Evidencias de las acciones de los niños

Unidad didáctica integrada: "El Circo"

Integración de campos del pensamiento: comunicación, arte y expresión, histórico-social, científico y matemático

\begin{tabular}{|c|c|c|}
\hline Concreción curricular & Transposición didáctica & $\begin{array}{l}\text { Valoración de lo } \\
\text { aprendido }\end{array}$ \\
\hline $\begin{array}{l}\text { Derechos básicos de aprendizaje } \\
\text { Flexibilización curricular }\end{array}$ & $\begin{array}{l}\text { La concreción curricular se } \\
\text { adquiere por medio de TAREAS } \\
\text { (ACTOS). }\end{array}$ & $\begin{array}{l}\text { Autoevaluación. } \\
\text { Coevaluación. } \\
\text { Heteroevaluación. }\end{array}$ \\
\hline $\begin{array}{l}\text { Formas de pensar: } \\
\text { 1.Desarrolar habilidades de pensamiento como la } \\
\text { observación, la clasificación y la comparación. } \\
\text { 2. Favorecer el desarrollo de habilidades de } \\
\text { pensamiento matemático como el razonamiento, } \\
\text { la resolución de problemas, la ubicación espacio } \\
\text { temporal, el pensamiento geométrico y la } \\
\text { modelación. } \\
\text { 3. Relacionar conceptos básicos de los campos de } \\
\text { pensamiento en el contexto de la pandemia a través } \\
\text { de la integración de los distintos saberes. }\end{array}$ & $\begin{array}{l}\text { Se concreta en ACTIVIDADES } \\
\text { que incluyen unos EJERCICIOS. } \\
\text { Todo se desarrolla a través de } \\
\text { una METODOLOGİA. } \\
\text { Instrucción directa simulación en } \\
\text { un ESCENARIO DIDÁCTICO. } \\
\text { En casa MATERIALIZACIÓN } \\
\text { Cartilla. }\end{array}$ & $\begin{array}{l}\text { Intervención directa } \\
\text { del producido para } \\
\text { realimentación. } \\
\text { Lista de chequeo. } \\
\text { Fotos y videos. } \\
\text { Páginas interactivas } \\
\text { de evaluación. }\end{array}$ \\
\hline $\begin{array}{l}\text { Herramientas para ser parte del mundo } \\
\text { 4. Oralidad, lectura y escritura: ejercitación de la } \\
\text { conciencia fonológica, la conciencia fonética, la } \\
\text { conciencia semántica y la conciencia sintáctica, } \\
\text { cuando lee, comprende, transcribe, toma dictado y } \\
\text { escribe diversos textos. }\end{array}$ & & \\
\hline
\end{tabular}

Fuente: Elaboración propia. 


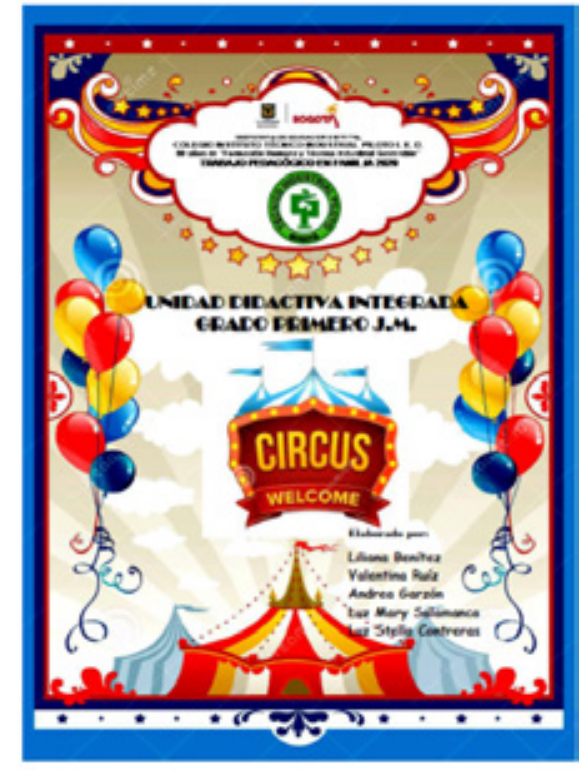

20

Figura 2. Cartilla pedagógica.

Fuente: Archivo personal de los autores.

En este sentido, la planificación curricular de la unidad didáctica integrada, como una forma de "[...] programación y actuación docente" (Ander-Egg, 2018), organizó e incluyó lo siguiente:

1. Actividades para desarrollar en la cartilla pedagógica: apoyadas en materiales para recortar, audios, vídeo, imágenes recortables, paginas interactivas, entre otras.

2. Rutina: direccionada para que los niños y niñas trabajaran en su cuaderno; esta contiene unas acciones que siempre debían seguir los niños, para dar una organización a los cuadernos, dado que ellos estaban iniciando su proceso lecto-escritor y era necesario el uso y manejo del cuaderno, así como de los trazos, escritura del nombre y familiarización del uso de mayúsculas y minúsculas.

3. Actividades para evaluar: apoyadas en páginas interactivas.

4. Actividades de extensión o para reforzar: susceptibles de realizarse de manera optativa, en tanto no todos los niños y niñas tienen la posibilidad de la conectividad para acceder a ellas.

5. La hora de evaluar: también se establece como una rutina en la que el niño, si está trabajando en la cartilla, tendrá dos recuadros, uno en donde se autoevaluará con una "carita", y otro, para la coevaluación, donde el padre de familia evaluará el proceso académico del niño, al ser su acompañante; proceso que, igualmente debía hacerse, cuando el trabajo era en el cuaderno, basado en unos criterios comunicados con antelación para padres de familia y estudiantes.

6. El proceso de heteroevaluación: se realiza mediante el envió de fotos en formato PDF que sirven como evidencias de la realización de las actividades de la cartilla y del cuaderno. El maestro interviene las actividades con sus observaciones y realimentación, cuyo objeto es ir mejorando los procesos académicos planteados.

Como conclusión de este ejercicio, es importante resaltar que la situación de pandemia puso a prueba, como ocurría generalmente en la presencialidad, la capacidad de reflexión y la producción pedagógica, metodológica y didáctica de los maestros. Y con esto, su capacidad creadora y recreadora para producir, en colectivo, diversas alternativas de trabajo pedagógico que procuraron darle continuidad a la 
educación, favoreciendo los aprendizajes y la producción de conocimientos, que le sirvieran a los niños para comprender la complejidad del mundo en que viven.

\section{Las guías integradas}

El año 2020, un tiempo de pandemia mundial, provocó la movilización de las dinámicas escolares, educativas y pedagógicas, en un escenario diferente al espacio físico del aula. Esto significó incorporar y establecer maneras distintas de enseñar los saberes académicos y de promover el aprendizaje estudiantil, como también maneras de relacionarse con la comunidad educativa, convirtiéndose la comunicación en el vehículo esencial del proceso de enseñanza y aprendizaje (Amayuela et. al., 2005), a menudo, apoyada por el empleo de herramientas virtuales y digitales. En este sentido, el reto para los maestros y estudiantes, particularmente, los de grado segundo del colegio Entre Nubes Sur Oriental, sede C, Ca- nadá Güira, era el de superar esas dificultades que el distanciamiento y el confinamiento les proponía. Ante esto, los maestros se vieron obligados a rediseñar sus formas de enseñanza; los estudiantes a mantener su compromiso frente a sus procesos formativos; y la familia, a pesar de sus responsabilidades laborales, a asumir el rol de acompañar constantemente el ejercicio académico, y a compartir las responsabilidades educativas, supervisadas a la distancia por el maestro, pues es él, quien modifica el sistema educativo, lo siente y lo hace (Ferreyra, 2020).

Para mantener el contacto con los estudiantes y darle continuidad a la educación, se propuso el diseño de una cartilla pedagógica, constituida por guías integradas (Figura 4), que se desarrollarían quincenalmente en casa, y en la que, cada profesor tuvo una participación específica, formulando las actividades didácticas para el grado, unificando en ellas, los contenidos, los criterios de evaluación y los requerimientos de aprendizaje esenciales de los estudiantes.

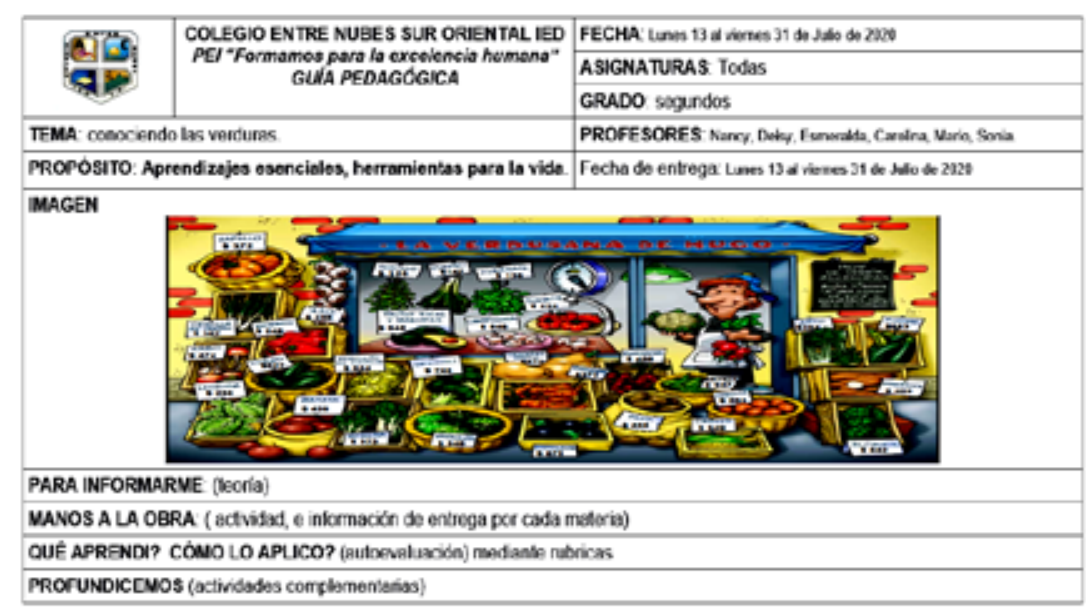

Figura 3. Modelo de la guía integrada.

Fuente: Archivo personal de los autores. 
Gracias a este ejercicio, se mantuvo en gran medida, el trabajo colectivo de los maestros frente al diseño de las guías integradas, en espacios mediados, en los que se deliberó, discutió y validó el trabajo pedagógico realizado. Además, se negoció y aprendió de las formas de enseñanza de los otros maestros, de sus posturas educativas, didácticas, pedagógicas y epistemológicas. Por ello, fue posible conocer, dar cuenta de lo que se privilegia al enseñar, aprender e interrogar las prácticas educativas, dilucidar las diferentes estrategias pedagógicas que se utilizan y los estilos de evaluación de los aprendizajes empleados por los maestros.

Así, el diseño y elaboración de la cartilla pedagógica, se convirtió en un espacio de encuentro pedagógico, en el que se interactuó para decidir lo que los estudiantes debían aprender y conocer en tiempos de pandemia. Dicho material se compartió a los estudiantes por diversos medios (digitales y en físico), buscando brindar una "educación para todos" y dar continuidad a la estrategia de "Aprender en casa”. Es importante destacar que la irrupción de la cartilla pedagógica en los hogares de los estudiantes trastocó la privacidad que tenían las familias y los maestros. A pesar del distanciamiento, se hizo constante la comunicación a través de diversos medios como la llamada a celular o el mensaje por WhatsApp, en cualquier horario del día. La vida familiar de los estudiantes y la vida de los maestros se hicieron públicas. Las historias, las expectativas, las necesidades, los deseos e intereses se compartieron; el confinamiento acercó más la escuela a la casa y los hogares irrumpieron a través de las pantallas en la existencia de los maestros.
En conjunto, los medios y plataformas virtuales se configuraron en escenarios para el aprendizaje, para la resolución de las dudas e inquietudes respecto a las actividades planteadas en la cartilla, realizando un esfuerzo comunicativo que implicó determinar lo que provocan las palabras en quien las recibe (Mazza, 2020), para determinar los progresos estudiantiles, en una perspectiva digital. Igualmente, fue un espacio para resolver no solo las cuestiones pedagógicas, sino para atender asuntos institucionales, pues allí, se asignaron tareas y responsabilidades a los maestros, en un intento de perpetuar la escuela en las redes sociales. Un reto de por sí complejo, ya que la escuela se constituyó para ser presencial y permitir el contacto físico y directo con los demás, donde la producción de conocimiento, el desarrollo humano y el aprendizaje, se logran en gran medida mientras se asista a ella.

Ahora bien, para este año, ante las vivencias del año anterior, se modificó la estructura de la cartilla pedagógica con el fin de asumir la forma de un módulo de actividades (Figura 5), en el que nuevamente se formularon los contenidos, los objetivos, los aprendizajes, las competencias a desarrollar, las actividades a realizar por parte de los estudiantes y los criterios de evaluación; todo este material se sustentó en los encuentros sincrónicos y asincrónicos para garantizar la continuidad educativa. Este material, al igual que la cartilla pedagógica, se concibió como el resultado de un trabajo colectivo, participativo, deliberativo y reflexivo de los maestros de cada uno de los grados. 


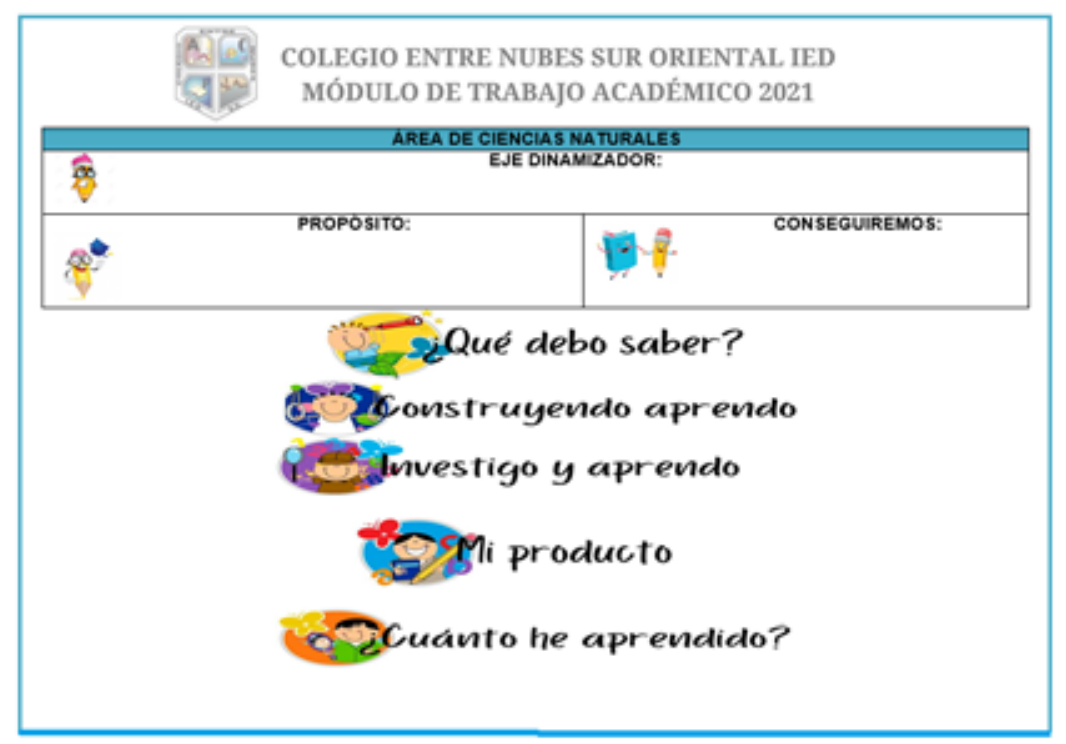

Figura 4. Formato de módulo.

Fuente: Archivo personal de los autores.

Con el diseño y la entrega del módulo de actividades, se procuró mantener la flexibilización de la enseñanza, ajustando las estrategias didácticas a las necesidades, expectativas e intereses de los estudiantes, así como la adecuación de los criterios de evaluación y la valoración del acompañamiento en casa y en la virtualidad del maestro. Esto permitió visibilizar el valor y sentido de la escuela pública (y por supuesto, del maestro) como un espacio en el que, a pesar de la virtualidad, se brindan oportunidades e intenta responder y garantizar la continuidad educativa, los aprendizajes y la formación de los estudiantes.

Como se advirtió, la condición de la pandemia se convirtió en una oportunidad para integrar de un modo más marcado, el mundo digital a la enseñanza, a la escuela y al maestro. Sin embargo, no para reemplazarla ni diluirla. Al contrario, para ofrecerle unos medios distintos de información y conocimiento, así como de encuentro distinto al presencial. A su vez, significó una posibilidad para el maestro de continuar con los procesos de formación y actualización pedagógica. Particularmente, se logró una comunicación más constante entre los integrantes del equipo pedagógico "Maestros en Colectivo" para compartir e intercambiar sentimientos, expectativas, intereses y experiencias en pandemia; dar continuidad a la elaboración de artículos en revistas y participar en encuentros nacionales, como "Pandemia y Escuela en Bogotá. Crónicas de maestras y maestros, 2020”, auspiciado por el IDEP, e internacionales, como el IV Congreso Internacional Dokuma "Redes, colectivos, nodos y semilleros de la SED". 


\section{Abstracción Geométrica: estrategia didáctica que se moviliza en tiempos de pandemia}

Antes de declararse la situación de pandemia mundial, uno de los trabajos que venía circulando en el Colegio Entre Nubes S. O., era la propuesta "Abstracción Geométrica: estrategia didáctica para el desarrollo del pensamiento geométrico de los niños en la escuela primaria", una alternativa de trabajo en el aula, que surgió en el año 2013, cuyo objetivo fundamental era el desarrollo del pensamiento geométrico de los niños de básica primaria. Conceptualmente, desarrolló las categorías de análisis y razonamiento geométrico, apoyándose en el pensamiento de autores como Berthelot y Salin (1994), Broitman (2000), Fripp y Varela (2012), Hoffer (1990), Itzcovich, (2005), Quaranta, y Ressia de Moreno (2009) y Sadovsky, (1998); en cuanto a los procesos de pensamiento básico, se basó en autores como De Sánchez (1991, 2002). Metodológicamente, recurrió al diseño y ejecución de la unidad didáctica "Los elementos básicos de la geometría: observación, relación, análisis y resolución", la cual estaba constituida por una serie de actividades de enseñanza que, enmarcadas en la abstracción geométrica, procuraron el desarrollo del pensamiento geométrico, acercando a los niños al estudio de la geometría y a la construcción de una imagen matemática del espacio distinta.

A raíz de la condición contemporánea de la pandemia, dicha estrategia didáctica tuvo que realizar una serie de transformaciones que le permitieron mantenerse y ser viable durante este tiempo, acompa- ñando los procesos de desarrollo del pensamiento geométrico de los niños, empleando los dispositivos tecnológicos y los medios digitales. Entre esos cambios que enfrentó la estrategia didáctica, estuvieron, precisamente, el uso de los mecanismos, medios y herramientas que ofrecía el mundo digital, a fin no solo de compartir las actividades de enseñanza constitutivas de la unidad didáctica y mantener la comunicación y el contacto, sino dar continuidad al proyecto mismo y sus intenciones. Entre las mediaciones que se utilizaron se recurrió, por ejemplo, al empleo del correo electrónico (institucional) de los niños y el WhatsApp de los padres de familia.

Junto a esto, las actividades geométricas de la unidad didáctica se rediseñaron y acomodaron a un lenguaje más sencillo, susceptible de comprenderse tanto por los estudiantes como por los padres de familia, acudientes o cuidadores, al momento de realizarse, con lo que se esperaba que el acompañamiento fuese efectivo. Este ajuste significó la modificación de la estructura original de las actividades de enseñanza, pues, la estructura previa contenía una actividad de carácter preliminar, otra de desarrollo, y una final de profundización. Con la pandemia, se dejó solamente la actividad preliminar y la actividad de desarrollo para facilitar el ejercicio de acompañamiento en casa y su efectiva realización (Figura 6), conservando la rigurosidad del trabajo y el compromiso con el proyecto. Así las cosas, la unidad didáctica transformó el mecanismo de transmisión de la información contenida en las actividades, ajustándose el número de acciones a realizar y empleándose un lenguaje comprensible para los niños y padres de familia, que requirieran un acompañamiento virtual del maestro. 
Fecha:

Actiridad preliminar: Abstracción Geométrica

Observa la siguieate imagen que corresponde a un cuadro del pintor Kasimir Nalevich y resuelve las preguntas en el cuadermo de Natematicas:

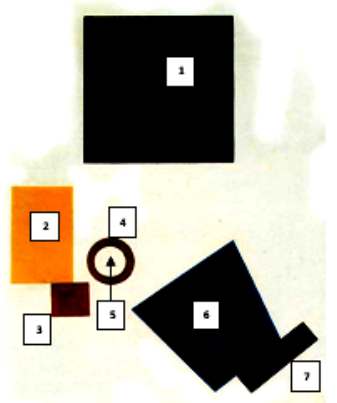

Actiridad de desarrollo:

A. Escribe los nombres de cada una de las figuras geométricas:

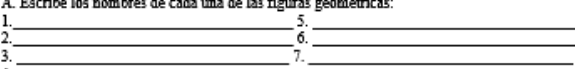

B. En qué se parecen las figuras geométricas representadas con los numeros 1 y 6 ? Menciona 3 carscterísticas.

C. Reaponde Falso (F) $\odot$ Verdadero (V) segin corresponda:

La fivira 1 y 3 son $C$

TERMINADA LA ACTIVDDAD ENVIAR IMAGEN EVIDENCIA (FOTO O PDF) DE LA ACTIVDAD REALIZADA, ;MUCHAS

Figura 5. Actividad de enseñanza ajustada.

Fuente: Archivo personal de los autores.

Estos mismos medios digitales, se emplearon para adelantar los procesos de calificación y evaluación de las actividades realizadas por los niños, lo que significó, para los padres de familia, aprender a tomar las fotos de las evidencias del trabajo de sus hijos y realizar su respectivo envío; y para el maestro, la conversión de estas imágenes al formato PDF, con el fin de retroalimentar lo realizado en casa (Figura 7). Gracias a este ejercicio, se fortaleció tanto el acercamiento y el desarrollo de capacidades relacionadas con el mundo virtual, como la aproximación a los avances de los estudiantes, en relación con su razonamiento geométrico. Es decir, realizar un proceso evaluativo de sus capacidades para desarrollar habilidades visuales, verbales, gráficas, aplicadas y lógicas, así como las de pensamiento, observación, comparación, descripción, entre otras.
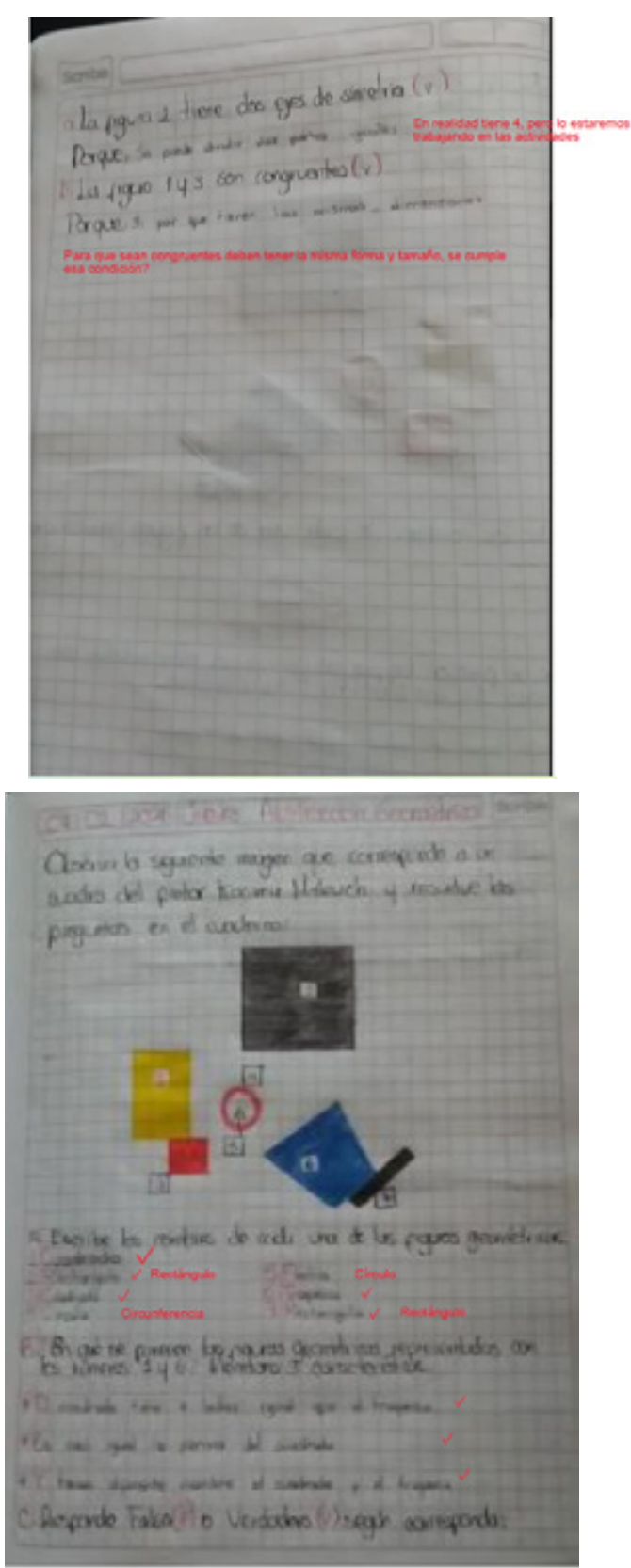

Figura 5. Actividad de enseñanza evaluada. Fuente: Archivo personal de los autores. 
No obstante, el acompañamiento familiar en la realización de las actividades de enseñanza, y el diseño de las mismas de una manera más comprensible por parte del maestro, hubo ocasiones en las que se presentaron situaciones en las que fue necesaria una explicación sobre el tema, para lo cual, nuevamente se recurrió a los medios de comunicación y contacto establecidos, como, por ejemplo, una videollamada por WhatsApp; de esta manera, se realizaron de modo sincrónico, acercamientos con los niños para resolver las dudas e inquietudes surgidas.

Es preciso aclarar, que a pesar del uso de este tipo de medios digitales y del acompañamiento de los padres de familia, la certificación de los aprendizajes y progresos de los niños, en relación con el desarrollo de su pensamiento geométrico, solamente era factible de validar a través de las actividades realizadas, en un ejercicio de observación indirecta.

Ahora bien, la estrategia didáctica no solo se transformó en estos aspectos, sino que mantuvo algunos elementos característicos de su estructura y organización. Entre ellos, sus intenciones pedagógicas, asociadas al desarrollo del razonamiento geométrico de los niños, en términos del progreso de las habilidades geométricas -visuales, verbales, gráficas, aplicadas y lógicas- establecidas por Hoffer (1990), así como sus procesos de pensamiento básico -observación, descripción, comparación, relación, clasificación, análisis, conceptualización, entre otros-, a través de las actividades de enseñanza constitutivas de la unidad didáctica: "Los elementos básicos de la geometría: observación, relación, análisis y resolución". Con esto, se buscó mantener la relación con el conocimiento geométrico, y por supuesto, con el desarrollo del pensamiento geométrico de los niños.
Este tipo de ejercicios, permitieron develar el interés y compromiso del maestro de movilizar su trabajo con el aprendizaje y desarrollo de los niños, como también con la producción de saber, a pesar del distanciamiento y del manejo de los medios virtuales como condición sine qua non para darle continuidad a la educación y a los procesos formativos en casa.

Conforme con las transformaciones y permanencias, se dieron unos desvanecimientos en la propuesta. Es decir, se abandonaron ciertas prácticas y maneras de intervenir, que hacía parte del trabajo pedagógico en el aula. Esencialmente, la pérdida del contacto con los niños, pues debido a ello, se hizo más complejo hacer el acompañamiento, proporcionar las indicaciones y conceptualizaciones exigidas, la aclaración de las dudas, la explicación de las acciones a ejecutar y la evaluación del modo cómo se realizan en cada momento las actividades de enseñanza formuladas. Y es que precisamente esta interacción sin la mediación de la pantalla es la que permite que el maestro evidencie, de forma directa y en tiempo real, los progresos de los estudiantes en términos de desarrollo del pensamiento geométrico. Además, el maestro en la presencialidad, puede realizar una evaluación del proceso formativo estudiantil, generar adecuaciones y ajustes al trabajo formulado, que, en definitiva, posibilitan el enriquecimiento y realimentación de la propuesta.

Como se ve, la situación de pandemia actual le permitió al maestro acceder a otras mediaciones para proseguir con sus procesos pedagógicos. Más allá de emerger el mundo virtual como una acción para mejorar la calidad de la educación, cómo en su momento muchos discursos lo expusieron, el acceso a 
las herramientas digitales le proporcionó al maestro un formato distinto, un canal diferente de transmisión de la información y un medio posibilitar la continuidad de la educación de los estudiantes. El interés y las intenciones de los maestros, así como la preocupación por difundir las actividades de enseñanza y los proyectos pedagógicos en las redes, se mantuvieron en gran medida, porque el maestro es un sujeto productor de saber y un intelectual preocupado por el enriquecimiento del campo de la pedagogía.

\section{Reflexiones finales}

La descripción de estas tres experiencias pedagógicas sirven para determinar que lo vivido en tiempos de pandemia, contribuyó en gran medida, a resaltar el papel fundamental del maestro y la escuela en la formación educativa de los niños, niñas y jóvenes, que no es otro que determinar aquello que permanece desde el compromiso de los maestros con darle continuidad a la educación y los aprendizajes. A su vez, está lo que se transforma; es decir, la adopción de la virtualización del quehacer pedagógico, que dejó ver el compromiso, creatividad e interés de los maestros por mantener y darle continuidad a la educación en los hogares, lo cual, implicó la transfiguración del trabajo pedagógico, no solo en términos de la flexibilización de la enseñanza y de los ajustes a las acciones de intervención escolar sino la adopción de los medios digitales para mantener la formación subjetiva. Por eso, más allá de pretender que estas mediaciones virtuales reemplacen, "reinventen" o diluyan a la escuela y al maestro, la condición de la pandemia develó su importancia y necesidad en la formación humana y en el establecimiento de interacciones sociales.

Y, por último, lo que se desvanece. A saber, la intervención directa del maestro sobre los procesos educativos y evaluativos, así como la posibilidad de contacto inmediato con los otros agentes educativos para el ejercicio de la interacción social. 道 


\section{Referencias}

Amayuela, G., Colunga, S., y Álvarez, N. (2005). Docencia universitaria y comunicación educativa. Contexto educativo revista digital de investigación y nuevas tecnologías, n. 36. www.contexto-educativo.com-ar/2005/3/nota-o6

Ander-Egg, E. (2018). Programación de aula. https:// nanopdf.com/download/programacion-de-aula-ezequiel-ander-egg_pdf

Becerra Martínez, J. M., Cárdenas Forero, Ó. L., Uribe Garzón, S. M., Moncada Rodríguez, M. A., Negrete Soler, E. C., Prieto Acuña, A., y Garzón, J. J. (2021). Comunicación, educación y escuela en tiempos del covid-19: una mirada de maestros en colectivo. Educarnos. n. 40, pp. 101-114. https://revistaeducarnos.com/wp-content/uploads/2020/12/ james-frank.pdf

Berthelot, R., y Salin, M. (1994). La enseñanza de la geometría en la escuela primaria. Grand N. (53). Universidad de Bordeaux. Ministerio de Cultura y Educación. PTFD.

Broitman, C. (2000). Reflexiones en torno a la enseñanza del espacio. Educación Matemática. Propuestas de trabajo, experiencias y reflexiones. La educación en los primeros años. Ediciones Novedades Educativas.

CEPAL-Unesco (2020). Informe COVID-19. La educación en tiempos de la pandemia de COVID-19. https://doi.org/10.24875/j.gamo.M20000200
De Sánchez, M. (1991). Desarrollo de habilidades del pensamiento. Procesos básicos del pensamiento. Editorial Trillas,

De Sánchez, M. (2002). La investigación sobre el desarrollo y la enseñanza de las habilidades de pensamiento. Revista Electrónica de Investigación Educativa, v. 4, n. 1. https://redie.uabc.mx/redie/ article/view/55

Ferreyra, P. (30 de marzo de 2020). Cartas al País. La oportunidad del sistema educativo en tiempos de pandemia. Diario El Clarín. https://www.clarin. com/cartas-al-pais/oportunidad-sistema-educativo-tiempos-pandemia_0_1ij5Wp9oB.html

Fripp, A. y Varela, C. (2012). Pensar geometricamente. Actas del cuarto Congreso Uruguayo de Educación Matemática (CUREM 4), pp. 11-16. http://funes. uniandes.edu.co/17654/1/Fripp2012Pensar.pdf

Hoffer, A. (abril de 1990). La geometría más que una demostración. Notas de Matemática. 29, pp. 10-24. Universidad de Oregón.

Hurtado Talavera, F. J. (julio-agosto, 2020). La educación en tiempos de pandemia: los desafíos de la escuela del siglo XXI. CIEG. Revista arbitrada del Centro de Investigación y Estudios Gerenciales, v. 44, pp. 176-187. http://www.grupocieg.org/archivos_revista/Ed.44(176-187)\%20Hurtado\%20 Tavalera_articulo_id650.pdf 
Itzcovich, H. (2005). Iniciación al estudio didáctico de la geometría. De las construcciones a las demostraciones. Libros del Zorzal. https://juliobaigorria. files.wordpress.com/2016/04/iniciacic3b3n-al-estudio-didc3a1ctico-de-la-geometrc3ada-horacio-itzcovich.pdf

Maestros en Colectivo. (2021). Comunicación, educación y escuela en tiempos de COVID-19: Una mirada de Maestros en Colectivo. Alcaldía Mayor de Bogotá. IDEP. pp. 1-22. Bogotá.

Mazza, D. (2020). Lo que la pandemia nos deja: una oportunidad para pensarnos como docentes. Centro de Innovación en Tecnología y Pedagogía. UBA Académica. http://educaciondelamirada.com/ wp-content/uploads/2020/04/AcaDocs_D07_Loque-la-pandemia-nos-deja31628.pdf

Naciones Unidas (2020). Informe de políticas: La educación durante la COVID-19 y después de ella. https://www.un.org/sites/un2.un.org/files/policy_brief_-_education_during_covid-19_and_beyond_spanish.pdf
Quaranta, M. E. y Ressia de Moreno, B. (2009). La enseñanza de la geometría en el jardín de infantes. Serie desarrollo curricular. Dirección General de Cultura y Educación. http://abc.gob.ar/inicial/ sites/default/files/2_la_ensenianza_de_la_geometria_en_el_jardin_de_infantes.pdf

Sadovsky, P., Parra, C. Itzcovich, H. y Broitman, C. (1998). La enseñanza de la geometría en el segundo ciclo. Documento de actualización curricular $N^{\circ} 5$. Bs As: Dirección de Currícula. https://www.buenosaires.gob.ar/sites/gcaba/files/ep_ac_mate_ doc5.pdf

Secretaría de Educación del Distrito (SED). (2007). Orientaciones curriculares para el campo de Comunicación, Arte y Expresión. Colegios públicos de excelencia para Bogotá. Serie: Cuadernos de Currículo. Bogotá sin indiferencia. 